Article

\title{
Proteomic Analysis of Matched Formalin-Fixed, Paraffin-Embedded Specimens in Patients with Advanced Serous Ovarian Carcinoma
}

\author{
Ashlee L. Smith ${ }^{1, *}$, Mai Sun ${ }^{2}$, Rohit Bhargava ${ }^{3}$, Nicolas A. Stewart ${ }^{2}$, Melanie S. Flint ${ }^{4,5,6}$, \\ William L. Bigbee ${ }^{5}$, Thomas C. Krivak ${ }^{1,5,7}$, Mary A. Strange ${ }^{5}$, Kristine L. Cooper ${ }^{6}$ and \\ Kristin K. Zorn ${ }^{1,5,7}$
}

1 Division of Gynecologic Oncology, Magee-Womens Hospital of UPMC, Pittsburgh, PA 15213, USA; E-Mails: tomkrivak@verizon.net (T.C.K.); kkzorn@aol.com (K.K.Z.)

2 Biomedical Mass Spectrometry Center for the Health Sciences, University of Pittsburgh, Pittsburgh, PA 15213, USA; E-Mails: sunm3@upmc.edu (M.S.); nas96@pitt.edu (N.A.S.)

3 Department of Pathology, University of Pittsburgh School of Medicine, Pittsburgh, PA 15213, USA; E-Mail: rbhargava@mail.magee.edu

4 Departments of Pharmacology and Chemical Biology, University of Pittsburgh School of Medicine, Pittsburgh, PA 15213, USA; E-Mail: flintms@upmc.edu

5 Women's Cancer Research Center, Pittsburgh, PA 15213, USA; E-Mails:

bigbeew1@upmc.edu (W.L.B); mstrange@mwri.magee.edu (M.A.S.)

6 University of Pittsburgh Cancer Institute, Pittsburgh, PA 15213, USA;

E-Mail: cooperk3@upmc.edu

7 Magee-Womens Research Institute, Pittsburgh, PA 15213, USA

* Author to whom correspondence should be addressed: E-Mail: alsmith1@geisinger.edu; Tel.: +1-570-271-6064; Fax: +1-570-271-5819.

Received: 25 July 2013; in revised form: 8 October 2013 / Accepted: 8 October 2013 / Published: 17 October 2013

\begin{abstract}
Objective: The biology of high grade serous ovarian carcinoma (HGSOC) is poorly understood. Little has been reported on intratumoral homogeneity or heterogeneity of primary HGSOC tumors and their metastases. We evaluated the global protein expression profiles of paired primary and metastatic HGSOC from formalin-fixed, paraffin-embedded (FFPE) tissue samples. Methods: After IRB approval, six patients with advanced HGSOC were identified with tumor in both ovaries at initial surgery. Laser capture microdissection (LCM) was used to extract tumor for protein digestion. Peptides were extracted and analyzed by reversed-phase liquid chromatography coupled to a linear
\end{abstract}


ion trap mass spectrometer. Tandem mass spectra were searched against the UniProt human protein database. Differences in protein abundance between samples were assessed and analyzed by Ingenuity Pathway Analysis software. Immunohistochemistry (IHC) for select proteins from the original and an additional validation set of five patients was performed. Results: Unsupervised clustering of the abundance profiles placed the paired specimens adjacent to each other. IHC H-score analysis of the validation set revealed a strong correlation between paired samples for all proteins. For the similarly expressed proteins, the estimated correlation coefficients in two of three experimental samples and all validation samples were statistically significant $(p<0.05)$. The estimated correlation coefficients in the experimental sample proteins classified as differentially expressed were not statistically significant. Conclusion: A global proteomic screen of primary HGSOC tumors and their metastatic lesions identifies tumoral homogeneity and heterogeneity and provides preliminary insight into these protein profiles and the cellular pathways they constitute.

Keywords: serous ovarian carcinoma; proteomics; laser capture microdissection

\section{Introduction}

Epithelial ovarian cancer (EOC) is the most lethal gynecologic malignancy [1]. The majority of patients with EOC present with metastatic lesions identified at primary surgery and experience recurrence. This type of clinical behavior affords researchers the opportunity to evaluate multiple tumor samples throughout a patient's clinical course.

The biology of high-grade serous ovarian carcinoma (HGSOC) and its metastases remains poorly understood [2,3]. Serous carcinomas are the most common subtype of EOC and are further divided into low-grade and high-grade based on molecular and morphologic features. Low-grade serous ovarian carcinoma is frequently associated with mutations in $B R A F$ and $K R A S$, demonstrates mild to moderate nuclear atypia, and often expresses higher levels of estrogen receptors, progesterone receptors, and E-cadherin [3]. In contrast, HGSOC is defined by ubiquitous TP53 mutations, defects in homologous DNA repair with chromosomal instability, and high cellular proliferation [2]. Given this genomic instability, the molecular features of HGSOC vary widely across tumors (intertumoral heterogeneity) but also within tumors (intratumoral heterogeneity) [2,4]. The role of tumoral heterogeneity in treatment response and recurrence patterns is not clearly defined.

Multiple high-throughput research techniques have been applied in studying HGSOC. Attempts to identify serum biomarkers, investigations of the histologic types, and genomic studies of metastatic lesions have been completed utilizing fresh-frozen samples [5-9]. However, extensive fresh-frozen tissue collections are rare. Formalin-fixed, paraffin-embedded (FFPE) tissue is the most common source for archived pathologic specimens. FFPE tissue collections have become important resources for studies of DNA, RNA, and more recently, intact proteins [10-12].

Proteomics involves the large-scale identification, characterization, and quantitation of proteins expressed in a tissue [13]. An advantage of proteomics is the ability to examine the biochemical and cellular phenotypic states, rather than genotypic expression patterns [14]. Mass spectrometry applications 
have been used to look for biomarkers in both the ascites and serum of EOC patients [15-17]. Additionally, studies have evaluated histologic subclassifications of EOC, characterized tumors by stage of disease, and analyzed samples of HGSOC for markers of chemosensitivity and chemoresistance [17-21]. Initially, proteomic studies utilized HGSOC tissue sources ranging from fresh-frozen specimens to ovarian cancer cell lines [13,22]. In 1998, Ikeda et al. described the extraction of intact protein from FFPE tissues [23]. Currently, faster and more efficient techniques have been developed to allow the extraction of full-length, non-degraded proteins from FFPE tissues [24-26]. Coupling abundant FFPE samples with laser capture microdissection (LCM) technology permits the dissection of particular areas of interest. Additionally, while access to fresh-frozen primary tumors and matched metastatic sites is often limited, matched FFPE tissues are readily available.

To date, the proteomic profiles of primary and metastatic EOC at the time of initial debulking surgery have not been investigated. Proteomic analysis across different tissue sites (for example, omentum and ovary) can be complicated by the inability to distinguish the inherent background differences in protein expression between the sites from the differences that are due to tumor expression in each site. In this study, our goal was to evaluate the protein expression profiles via a global proteomic screening of HGSOC from FFPE samples of both ovaries, with the presumption that one side represents the primary tumor and the other side a metastatic site, therefore eliminating heterogeneity among different tissue types. Following identification, selected proteins involved with migration, cell adhesion, and metastasis were investigated using IHC to assess the correlation of findings.

\section{Experimental}

\subsection{FFPE Collection, Processing for Proteomics}

Following approval from the University of Pittsburgh IRB, FFPE samples from six patients with International Federation of Gynecology and Obstetrics Stages III or IV HGSOC and tumor present in both ovaries at the time of initial surgery were identified as the experimental set of patients (E-HGSOC). Five additional patients (V-HGSOC), matched for age and stage, were identified and used in addition to the original six patients for evaluation by IHC. All patients underwent initial debulking surgery at Magee-Womens Hospital of UPMC. Hematoxylin and Eosin (H\&E) stained tissue specimens were evaluated by a gynecologic pathologist to confirm the diagnosis and presence of tumor in both ovaries. Tissue samples were cut by microtome to $10 \mu \mathrm{m}$ sections and placed onto laser microdissection slides (Leica Microsystems, Inc., Bannockburn, IL, USA). Slides were lightly stained via standard H\&E protocol immediately prior to completion of LCM. Cancer cells from defined regions of the ovaries were acquired by laser microdissection (Leica LMD 6000, Leica Microsystems, Inc.) and collected in $40 \mu \mathrm{L}$ of purified water in RNase/DNase-free microcentrifuge tubes. Six million microns of tissue was the targeted amount to be collected. Samples were brought to $100 \mathrm{mM}$ $\mathrm{NH}_{4} \mathrm{HCO}_{3}, \mathrm{pH} \mathrm{8.4,20 \%}$ acetonitrile and incubated in a thermal cycler at $100{ }^{\circ} \mathrm{C}$ for $1 \mathrm{~h}$, and thereafter, $65{ }^{\circ} \mathrm{C}$ for an additional $2 \mathrm{~h}$. Samples were cooled to ambient temperature, followed by addition of $100 \mathrm{ng}$ of modified porcine sequencing grade trypsin (Promega Corporation, Madison, WI, USA) and incubated for $16 \mathrm{~h}$ at $37{ }^{\circ} \mathrm{C}$. Samples were vacuum-dried and desalted using PepClean desalting 
columns (Pierce, Rockford, IL, USA) according to the manufacturer's protocol. Eluted peptides were vacuum-dried and stored at $-80^{\circ} \mathrm{C}$.

\subsection{Liquid Chromatography Tandem Mass Spectrometry}

Peptide digests were resuspended in $0.1 \%$ TFA and analyzed in triplicate by reversed-phase liquid chromatography (RPLC)-tandem mass spectrometry (MS/MS) using an Ultimate 3000 Nanoflow LC (Dionex Corporation, Sunnyvale, CA, USA) coupled online to an LTQ-Orbitrap XL (ThermoFisher Scientific, San Jose, CA, USA). Separations were performed using $75 \mu \mathrm{m} \mathrm{ID} \times 360 \mu \mathrm{m}$ OD $\times 20 \mathrm{~cm}$-long fused silica capillary columns (Polymicro Technologies, Phoenix, AZ, USA), slurry-packed in house with $5 \mu \mathrm{m}, 300 \AA$ pore size C-18 silica-bonded stationary phase (Jupiter, Phenomenex, Torrance, CA, USA). Following sample injection onto the C-18 pre-column (Dionex), the column was washed for 3 min with mobile phase A ( $2 \%$ acetonitrile, $0.1 \%$ formic acid) at a flow rate of $30 \mu \mathrm{L} / \mathrm{min}$. Peptides were eluted using a linear gradient of $0.33 \%$ mobile phase B $(0.1 \%$ formic acid in acetonitrile $) / \mathrm{min}$ for $130 \mathrm{~min}$, then to $95 \% \mathrm{~B}$ in an additional $15 \mathrm{~min}$, all at a constant flow rate of $200 \mathrm{~nL} / \mathrm{min}$. Column washing was performed at $95 \%$ B for $15 \mathrm{~min}$ for all analysis, after which the column was re-equilibrated in mobile phase A prior to subsequent injections. The MS was operated in data-dependent MS/MS mode in which each full MS scan was collected in the Orbitrap, followed by seven MS/MS scans performed in the linear ion trap (LIT) where the seven most abundant peptide molecular ions were selected for collision-induced dissociation (CID), using a normalized collision energy of 35\%. Data were collected over a precursor ion range of 375-1,800 m/z $(\mathrm{R}=60,000 @ 400 \mathrm{~m} / \mathrm{z})$ and dynamic exclusion was enabled to minimize redundant selection of peptides previously selected for CID.

\subsection{Peptide Identification}

Tandem mass spectra were searched against the UniProt human protein database (10/11 release) from the European Bioinformatics Institute, using SEQUEST (BioWorks, v3.2, ThermoScientific) [27]. Search criteria were set as follows: peptides were searched fully-tryptic with up to two missed cleavage sites, dynamic modification of methionine oxidation (15.9949 Da) and static modification of cysteine carbamidomethylation $(57.0215 \mathrm{Da})$, precursor mass tolerance of $20 \mathrm{ppm}$ and fragment ion tolerance of $0.5 \mathrm{Da}$. Identified peptides were further screened using the Peptide Prophet algorithm included in the Trans-Proteomic Pipeline software package (V4.3 rev.1) [28,29].

\subsection{Spectral Count Analysis}

The qualitative differences in protein abundance between samples were derived by summing the total CID events that resulted in a positively identified peptide for a given protein accession across all samples (spectral counting), as previously described [30]. Proteins included for analysis were filtered using the criteria that a minimum peptide count of two or greater for a given protein was present in at least four of the samples analyzed. The relative protein abundance levels between the right and left ovaries within each patient were assessed by fold changes. Proteins were compared between the right and left ovaries for each patient and classified by qualitative analysis of their spectral counts as 
proteins which were similarly expressed (less than a two-fold change) or differentially expressed (greater than or equal to a two-fold change) between the paired ovarian samples [31-33].

\subsection{Ingenuity Pathways Analysis}

Proteins identified (using UniProt accession numbers) as similarly expressed (without a two-fold change in at least four of the paired sets) or differentially expressed (with a two-fold change in at least four of the paired sets) were entered into IPA software (Ingenuity Systems, Redwood City, CA, USA) for pathway analysis. Functional analysis of these proteins was performed utilizing the "Core Analysis" function in IPA.

\subsection{Immunohistochemical Staining}

Analysis of the IPA results, and review of established literature, resulted in the selection of the proteins evaluated via IHC. Antibodies selected included: CTNNB1 (BD Biosciences, San Jose, USA), CDC42 (Santa Cruz, Dallas, USA), PPP2R1A (Santa Cruz), ANXA1 (BD Biosciences), PHB (Sigma-Aldrich, St. Louis, USA), and PRDX1 (AbCam, Cambridge, UK) (Table S1). IHC was completed on the E-HGSOC and V-HGSOC paired sets. IHC of the 5-micrometer thick paraffin sections was performed on a Leica Bond immunostainer with Leica reagents (Buffalo Grove, IL, USA). Sections were deparaffinized and treated with heat-induced epitope retrieval solutions. The sections were incubated with the primary antibody, followed by the post primary antibody, and finally the Bond polymer. The sections were treated with a peroxide block and DAB. Sections stained for CDC42 were treated with an additional 5-min DAB enhancer step that was not included in the other antibody staining protocols. The sections were counterstained with hematoxylin. Washes separated each incubation step. Adequate immunoreactive tissue samples were used as positive controls. Negative controls consisted of omitting the primary antibody and replacing it with an antibody diluent solution.

The immunohistochemical staining was semi-quantified using the H-score method [34,35]. The $\mathrm{H}$-score is given as the sum of the percent staining multiplied by an ordinal value corresponding to the intensity level $(0=$ none, 1 = weak, 2 = moderate, 3 = strong). With four intensity levels, the resulting score ranges from 0 (no staining in the tumor) to 300 (diffuse intense staining of the tumor). The following cellular areas were scored for each antibody: cytoplasm for PHB, CDC 42 and PRDX1; nucleus and cytoplasm for ANXA1 and PPP2R1A; both membranes and cytoplasm for CTNNB1. A Gynecologic Oncology Pathologist scored the IHC staining and was blinded to the specimen sources.

Agreement of H-scores and percent staining between paired samples was estimated using Pearson's correlation coefficient; $95 \%$ confidence intervals were reported for the experimental and validation sets separately.

\section{Results}

Patients meeting selection criteria for the E-HGOC and V-HGOC matched-paired sets were identified. All patients had stage III or IV disease. Most patients were Caucasian, with a mean age range of 45-50 years old (Table 1). 
Table 1. Patient demographics.

\begin{tabular}{ccc}
\hline & E-HGSOC Specimens ${ }^{\mathbf{a}, * * * *}$ & V-HGSOC Specimens ${ }^{\mathbf{b}, *}$ \\
\hline $\begin{array}{l}\text { Age at Diagnosis (y) } \\
\text { Age Range }\end{array}$ & $(\mathrm{n}=6)$ & $(\mathrm{n}=5)$ \\
Race & $45-50$ & $45-50$ \\
$\quad$ Caucasian & 5 & 5 \\
African American & 1 & 0 \\
Stage & & \\
IIIB & 0 & 1 \\
IIIC & 4 & 4 \\
IV & 2 & 0 \\
\hline
\end{tabular}

\footnotetext{
${ }^{a}$ E-HGSOC: Experimental high-grade serous ovarian cancer specimens; ${ }^{b}$ V-HGSOC: Validation high-grade serous ovarian cancer specimens; * All patients underwent primary debulking surgery between 1998 and 2012 at a single institution. All carcinomas evaluated were high-grade serous epithelial ovarian carcinomas; ** E-HGSOC specimens were analyzed via proteomic analysis and IHC, whereas the V-HGSOC specimens were analyzed by IHC only and used for validation of the proteomic findings.
}

A total of six slides per disease site were prepared for LCM analysis. One to two slides from FFPE blocks of ovarian tissue yielded a mean of 7.11 micrograms from LCM. Complete results of the spectral counts obtained from MS can be found in the supplemental data (Table S2). After cursory filtering of the MS data described above, unsupervised hierarchical clustering of the protein abundance profiles consistently placed the original six paired specimens (E-HGOC) adjacent to each other, indicating that within each patient, gross aspects of tumoral homogeneity were observed (Figure 1). While globally there were similarities identified between the samples, areas of distinct heterogeneity were also noted.

IPA for the proteins classified as similarly expressed yielded scores of 19 or higher for the top five networks. For proteins classified as differentially expressed, the top five networks had IPA scores of 13 or higher. Among the differentially expressed proteins, the most statistically significant network contained 35 interacting proteins from our original proteomic analysis. Top canonical pathways identified for similarly expressed proteins involved EIF2 signaling, gluconeogenesis/glycolysis, and regulation of eIF4 signaling. Canonical pathways identified most frequently among differentially expressed proteins included EIF2 signaling, acute phase response signaling, and aminoacyl-tRNA biosynthesis (Table S3A,B).

Six proteins noted on the IPA pathways, including CTNNB1, CDC42, PPP2R1A, ANXA1, PHB, PRDX1, were selected for IHC evaluation. Three proteins each were selected from the lists of similarly expressed proteins (ANXA1, PHB, PRDX1) and differentially expressed proteins (CTNNB1, CDC42, PPP2R1A). IHC staining was completed on the E-HGSOC and V-HGSOC paired samples (Tables 2 and 3). The validation data set shows a strong correlation of the H-scores between the paired samples for all proteins, whereas the $\mathrm{H}$-score correlation for the experimental set shows greater variation. The estimated correlation coefficients in the validation samples for the similarly expressed proteins and two of the three estimated correlation coefficients for the experimental sample proteins classified as similarly expressed were statistically significant at the 0.05 level (Figure 2). However, the estimated correlation coefficients in the experimental sample proteins classified as differentially expressed were not statistically significant. 
Figure 1. Unsupervised hierarchical cluster analysis. After initial filtering of the proteins generated by MS, unsupervised hierarchal clustering analysis was completed which highlights the similar and different protein abundance profiles across the samples when compared to each other. Six proteins including CTNNB1, CDC42, PPP2R1A, ANXA1, PHB, PRDX1 were then selected for validation. The red-green color scheme indicates the rank normalized abundance of a protein relative to its average value across all six patients. (MATLAB script, MathWorks ${ }^{\circledR}$, Natick, MA, USA).

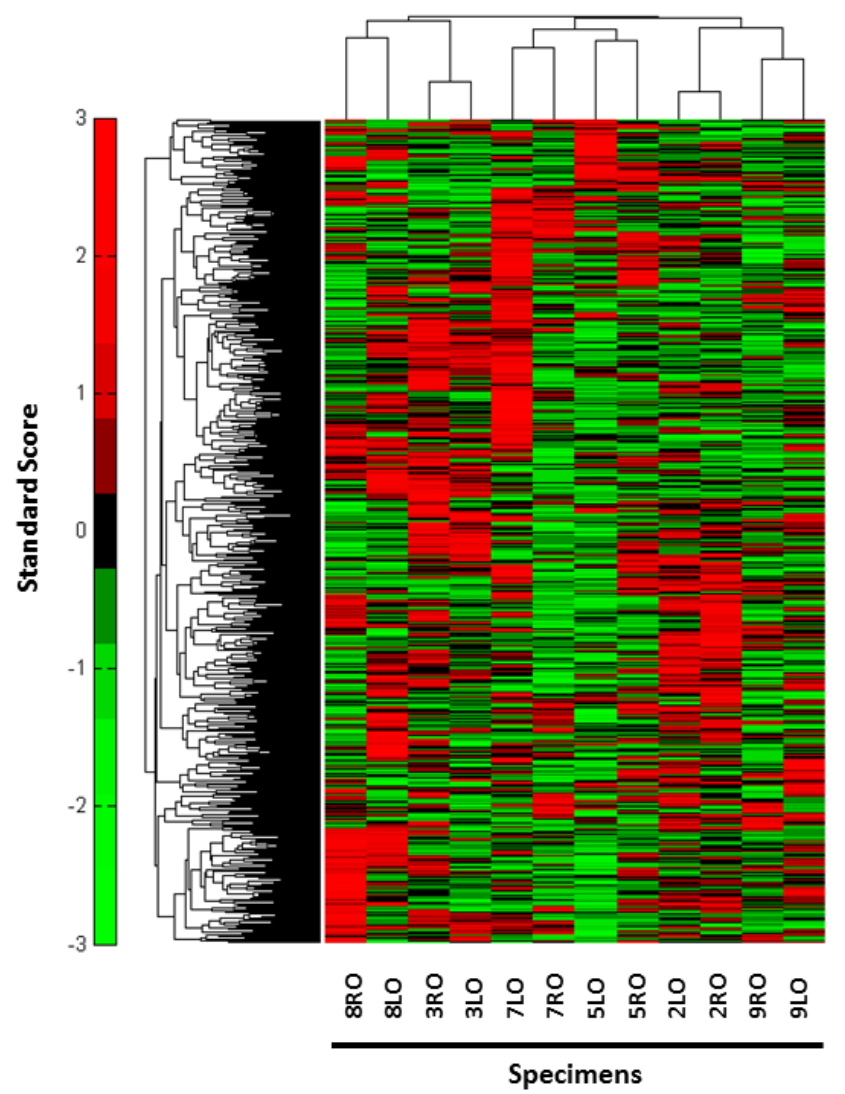

Table 2. Immunohistochemistry (IHC) table of results for antibodies representing proteins identified as having less than a two-fold change between paired samples.

\begin{tabular}{ccccccc}
\hline & \multicolumn{2}{c}{ PHB } & \multicolumn{2}{c}{ PDRX } & ANXA1 \\
\hline Sample & H-score & \% staining of cells & H-score & \% staining of cells & H-score & \% staining of cells \\
\hline E-HGSOC 2R & 115 & 70 & 120 & 80 & 280 & 95 \\
E-HGSOC 2L & 115 & 70 & 110 & 80 & 270 & 95 \\
E-HGSOC 3R & 120 & 70 & 20 & 20 & 260 & 90 \\
E-HGSOC 3L & 150 & 75 & 20 & 20 & 240 & 90 \\
E-HGSOC 5R & 170 & 90 & 40 & 30 & 240 & 95 \\
E-HGSOC 5L & 195 & 95 & 40 & 30 & 53 & 20 \\
E-HGSOC 7R & 185 & 95 & 20 & 20 & 265 & 95 \\
E-HGSOC 7L & 160 & 85 & 5 & 5 & 265 & 95 \\
E-HGSOC 8R & 105 & 60 & 5 & 5 & 260 & 100 \\
E-HGSOC 8L & 155 & 75 & 5 & 5 & 260 & 100 \\
E-HGSOC 9R & 165 & 90 & 20 & 20 & 170 & 60 \\
E-HGSOC 9L & 140 & 85 & 30 & 30 & 210 & 80 \\
\hline
\end{tabular}


Table 2. Cont.

\begin{tabular}{ccccccc}
\hline & \multicolumn{3}{c}{ PHB } & PDRX & ANXA1 \\
\hline Sample & H-score & \% staining of cells & H-score & \% staining of cells & H-score & \% staining of cells \\
\hline V-HGSOC 1R & 210 & 100 & 25 & 25 & 240 & 80 \\
V-HGSOC 1L & 210 & 100 & 25 & 25 & 270 & 90 \\
V-HGSOC 2R & 140 & 90 & 95 & 60 & 240 & 80 \\
V-HGSOC 2L & 165 & 90 & 120 & 70 & 210 & 70 \\
V-HGSOC 3R & 155 & 95 & 50 & 40 & 240 & 80 \\
V-HGSOC 3L & 150 & 80 & 75 & 50 & 240 & 90 \\
V-HGSOC 4R & 120 & 70 & 15 & 10 & 175 & 60 \\
V-HGSOC 4L & 130 & 90 & 15 & 15 & 175 & 60 \\
V-HGSOC 5R & 120 & 90 & 75 & 50 & 290 & 100 \\
V-HGSOC 5L & 130 & 90 & 75 & 50 & 283 & 98 \\
\hline
\end{tabular}

E-HGSOC: experimental high grade serous ovarian carcinoma samples; V-HGSOC: validation high grade serous ovarian carcinoma samples; The H-score is given as the sum of the percent staining multiplied by an ordinal value corresponding to the intensity level $(0=$ none, $1=$ weak, $2=$ moderate, $3=$ strong $)$. With four intensity levels, the resulting score ranges from 0 (no staining in the tumor) to 300 (diffuse intense staining of the tumor).

Table 3. IHC table of results for antibodies representing proteins identified as having a two-fold or greater change between paired samples (see the note of Table 2).

\begin{tabular}{ccccccc}
\hline & \multicolumn{2}{c}{ CTNNB1 } & \multicolumn{2}{c}{ CDC42 } & \multicolumn{2}{c}{ PPP2R1A } \\
\hline Sample & H-score & \% staining of cells & H-score & \% staining of cells & H-score & \% staining of cells \\
\hline E-HGSOC 2R & 55 & 50 & 60 & 60 & 150 & 90 \\
E-HGSOC 2L & 60 & 60 & 30 & 30 & 180 & 95 \\
E-HGSOC 3R & 155 & 80 & 60 & 60 & 110 & 60 \\
E-HGSOC 3L & 165 & 85 & 70 & 70 & 95 & 60 \\
E-HGSOC 5R & 160 & 100 & 100 & 80 & 160 & 90 \\
E-HGSOC 5L & 60 & 40 & 20 & 20 & 145 & 80 \\
E-HGSOC 7R & 140 & 85 & 60 & 60 & 125 & 90 \\
E-HGSOC 7L & 120 & 80 & 70 & 70 & 130 & 80 \\
E-HGSOC 8R & 90 & 60 & 50 & 50 & 90 & 70 \\
E-HGSOC 8L & 80 & 50 & 50 & 50 & 140 & 80 \\
E-HGSOC 9R & 185 & 95 & 40 & 30 & 115 & 70 \\
E-HGSOC 9L & 120 & 85 & 40 & 30 & 115 & 75 \\
V-HGSOC 1R & 150 & 100 & 90 & 90 & 100 & 70 \\
V-HGSOC 1L & 170 & 100 & 80 & 80 & 110 & 60 \\
V-HGSOC 2R & 180 & 100 & 80 & 80 & 95 & 75 \\
V-HGSOC 2L & 190 & 95 & 70 & 70 & 110 & 80 \\
V-HGSOC 3R & 130 & 80 & 70 & 70 & 100 & 70 \\
V-HGSOC 3L & 110 & 80 & 80 & 80 & 60 & 50 \\
V-HGSOC 4R & 200 & 100 & 70 & 70 & 90 & 70 \\
V-HGSOC 4L & 210 & 100 & 50 & 50 & 120 & 70 \\
V-HGSOC 5R & 210 & 100 & 50 & 50 & 40 & 40 \\
V-HGSOC 5L & 180 & 100 & 50 & 50 & 20 & 20 \\
\hline
\end{tabular}


Figure 2. Correlation of paired samples H-Scores across proteins.

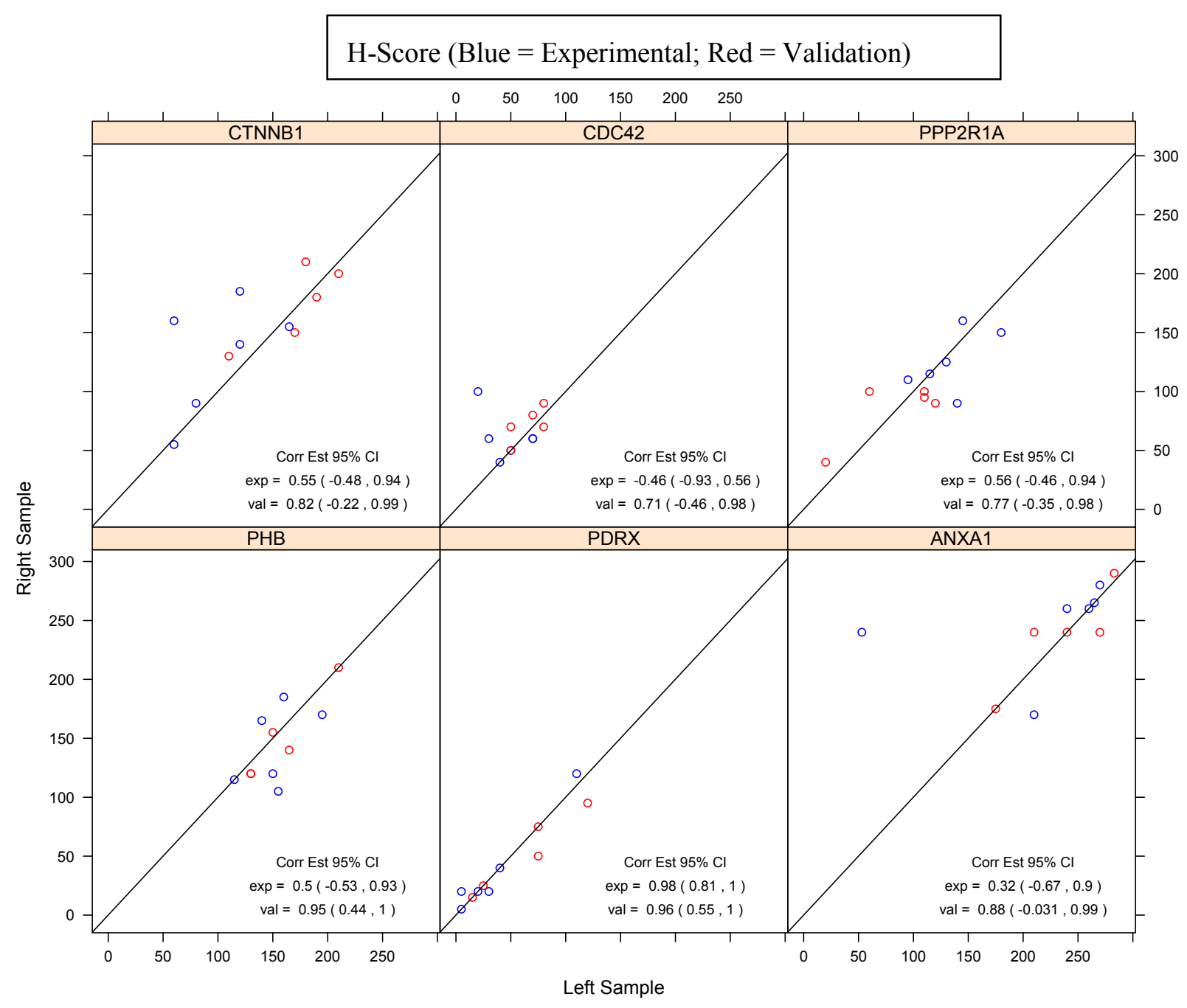

\section{Discussion}

Both intratumoral and intertumoral heterogeneity may play a significant role in tumor evolution, adaptation, and recurrence. This tumor variation can limit clinicians, who typically depend on the results of single tumor biopsies as they attempt to individualize treatment [4]. Better understanding of the biology of metastasis is critical, since we only rarely cure EOC when metastasis is present at diagnosis and are not able to cure recurrent EOC. The inherent complexity of genomic alterations between primary tumor and its metastases, coupled with numerous interactions between tumor and stromal cells, represent fundamental challenges in the quest to understand and control metastatic disease [14]. Comparisons of primary breast carcinomas with their corresponding metastatic brain lesions have demonstrated significant differences in the numbers of mutations found in a subset of cancer cells present in the primary lesion as compared to the metastatic site [36]. Genetic sequencing has now identified additional driver mutations in a subset of organ-specific metastases from pancreatic carcinoma that are not identified in the primary lesions [37]. These examples of key differences in the molecular biology of primary tumors and their metastatic lesions signal the potential difficulty of studying primary tumors as surrogates for what actually kills patients, their sites of metastatic spread. 
Herein, we elected to study primary and metastatic HGSOC within ovarian tissue. We analyzed six patients with advanced HGSOC with tumor present in both ovaries at their initial surgery to investigate the similarities and differences between these tumors. Ovarian tissue was used for comparison in an attempt to eliminate inherent protein expression differences among tissue sites, such as ovary, omentum, and lymph nodes. We integrated the techniques of LCM of FFPE specimens and mass spectrometry via discovery proteomics to assess the global proteomic profiles of these samples for similarly and differentially expressed proteins.

Proteins selected for evaluation via IHC were extrapolated from both the proteomic data obtained and their relevance in the literature, particularly those related to the concepts of heterogeneity and metastasis. PRDX1 has been identified as overexpressed in various subtypes of ovarian carcinoma and is implicated in the promotion and support of transformed tumor cells [38]. In prostate carcinoma specifically, PRDX family members are differentially expressed throughout various aspects of the tumor and have been suggested as a key family of proteins related to the disease process and response to treatment [39]. Underexpression of classes of annexins has been associated with chemoresistant disease and subsequently poor overall survival in ovarian carcinoma [18]. CDC42 and CTNNB1 are associated with cell-to-cell signaling and interaction, cellular assembly and organization, and tissue development. Both have demonstrated importance in the origination and continued propagation of disease in ovarian carcinoma and other solid tumors [40-42]. PPP2R1A has been identified as a key protein in the subclassifications of both serous endometrial and ovarian carcinomas, as well as clear cell carcinomas of the ovary. Mutations of this protein are linked to loss of negative regulation of cellular proliferation and loss of potential tumor suppression [43,44].

We recognize the small sample size for the original patients selected and the number of control cases as a limitation of our current study. However, this pilot project confirmed the feasibility of using LCM of FFPE tissue for discovery proteomics. Analysis with IHC did not correlate exactly with the proteomic findings in all cases. Perhaps our selected fold changes between samples were not significant enough to be detected using IHC methodology. Alternatively, spectral counting may not accurately quantitate these fold changes. Having completed the pilot attempt, additional patients can be studied. Using expanded results, further mechanistic avenues can be investigated to expand on the above noted concepts.

The complex biology of EOC remains a challenge for researchers trying to identify biomarkers for early diagnosis and clinicians treating patients with advanced disease. The advanced stage at disease presentation, varied individual responses to surgery and chemotherapy, and poor overall survival open many avenues for exploration in an attempt to better understand this disease. Identification of similarities and differences between primary tumors and their metastatic lesions on multiple structural levels may provide further insight into this challenging disease, and ultimately further our efforts to discovering a cure.

\section{Conclusions}

Proteomic analysis of HGSOC specimens from FFPE tissue is feasible, opening the door to further study with these samples that are well-annotated for clinical outcomes of interest. A global proteomic screen of primary HGSOC tumors and their metastatic lesions identifies tumoral homogeneity and 
heterogeneity, and provides preliminary insight into these protein profiles and the cellular pathways they constitute.

\section{Acknowledgments}

UPCI shared resources that are supported in part by award P30CA047904 were used for this project. We gratefully recognize the support of the Scaife Family Foundation and the Frieda G. and Saul F. Shapira BRCA Cancer Research Program. Zorn is also supported by NIH K12HD06308.

\section{Conflicts of Interest}

The authors declare no conflict of interest.

\section{References}

1. Siegel, R.; Naishadham, D.; Jemal, A. Cancer statistics, 2012. CA Cancer J. Clin. 2012, 62, 10-29.

2. Bromley, A.B.; Altman, A.D.; Chu, P.; Nation, J.G.; Nelson, G.S.; Ghatage, P.; Kalloger, S.E.; Han, G.; Kobel, M. Architectural patterns of ovarian/pelvic high-grade serous carcinoma. Int. J. Gynecol. Pathol. 2012, 31, 397-404.

3. Schmeler, K.M.; Gershenson, D.M. Low-grade serous ovarian cancer: A unique disease. Curr. Oncol. Rep. 2008, 10, 519-523.

4. Gerlinger, M.; Rowan, A.J.; Horswell, S.; Larkin, J.; Endesfelder, D.; Gronroos, E.; Martinez, P.; Matthews, N.; Stewart, A.; Tarpey, P.; et al. Intratumor heterogeneity and branched evolution revealed by multiregion sequencing. N. Engl. J. Med. 2012, 366, 883-892.

5. Cadron, I.; van Gorp, T.; Moerman, P.; Waelkens, E.; Vergote, I. Proteomic analysis of laser microdissected ovarian cancer tissue with SELDI-TOF MS. Methods Mol. Biol. 2011, 755, 155-163.

6. Tian, Y.; Yao, Z.; Roden, R.B.; Zhang, H. Identification of glycoproteins associated with different histological subtypes of ovarian tumors using quantitative glycoproteomics. Proteomics 2011, 11, 4677-4687.

7. Davidson, B. Anatomic site-related expression of cancer-associated molecules in ovarian carcinoma. Curr. Cancer Drug Targets 2007, 7, 109-120.

8. Espina, V.; Dettloff, K.A.; Cowherd, S.; Petricoin, E.F., 3rd.; Liotta, L.A. Use of proteomic analysis to monitor responses to biological therapies. Expert Opin. Biol. Ther. 2004, 4, 83-93.

9. Zorn, K.K.; Bonome, T.; Gangi, L.; Chandramouli, G.V.; Awtrey, C.S.; Gardner, G.J.; Barrett, J.C.; Boyd, J.; Birrer, M.J. Gene expression profiles of serous, endometrioid, and clear cell subtypes of ovarian and endometrial cancer. Clin. Cancer Res. 2005, 11, 6422-6430.

10. Klopfleisch, R.; Weiss, A.T.; Gruber, A.D. Excavation of a buried treasure-DNA, mRNA, miRNA and protein analysis in formalin fixed, paraffin embedded tissues. Histol. Histopathol. 2011, 26, 797-810.

11. Magdeldin, S.; Yamamoto, T. Toward deciphering proteomes of formalin-fixed paraffin-embedded (FFPE) tissues. Proteomics 2012, 12, 1045-1058.

12. Tanca, A.; Pagnozzi, D.; Addis, M.F. Setting proteins free: Progresses and achievements in proteomics of formalin-fixed, paraffin-embedded tissues. Proteomics Clin. Appl. 2012, 6, 7-21. 
13. Li, X.Q.; Zhang, S.L.; Cai, Z.; Zhou, Y.; Ye, T.M.; Chiu, J.F. Proteomic identification of tumor-associated protein in ovarian serous cystadenocarinoma. Cancer Lett. 2009, 275, 109-116.

14. Sethi, N.; Kang, Y. Unravelling the complexity of metastasis-Molecular understanding and targeted therapies. Nat. Rev. Cancer 2011, 11, 735-748.

15. Amon, L.M.; Law, W.; Fitzgibbon, M.P.; Gross, J.A.; O’Briant, K.; Peterson, A.; Drescher, C.; Martin, D.B.; McIntosh, M. Integrative proteomic analysis of serum and peritoneal fluids helps identify proteins that are up-regulated in serum of women with ovarian cancer. PLoS One 2010, 5, e11137.

16. Elschenbroich, S.; Ignatchenko, V.; Clarke, B.; Kalloger, S.E.; Boutros, P.C.; Gramolini, A.O.; Shaw, P.; Jurisica, I.; Kislinger, T. In-depth proteomics of ovarian cancer ascites: Combining shotgun proteomics and selected reaction monitoring mass spectrometry. J. Proteome Res. 2011, 10, 2286-2299.

17. Hoskins, E.R.; Hood, B.L.; Sun, M.; Krivak, T.C.; Edwards, R.P.; Conrads, T.P. Proteomic analysis of ovarian cancer proximal fluids: Validation of elevated peroxiredoxin 1 in patient peripheral circulation. PLoS One 2011, 6, e25056.

18. Kim, S.W.; Kim, S.; Nam, E.J.; Jeong, Y.W.; Lee, S.H.; Paek, J.H.; Kim, J.H.; Kim, J.W.; Kim, Y.T. Comparative proteomic analysis of advanced serous epithelial ovarian carcinoma: Possible predictors of chemoresistant disease. OMICS 2011, 15, 281-292.

19. An, H.J.; Kim, D.S.; Park, Y.K.; Kim, S.K.; Choi, Y.P.; Kang, S.; Ding, B.; Cho, N.H. Comparative proteomics of ovarian epithelial tumors. J. Proteome Res. 2006, 5, 1082-1090.

20. Bloom, G.C.; Eschrich, S.; Zhou, J.X.; Coppola, D.; Yeatman, T.J. Elucidation of a protein signature discriminating six common types of adenocarcinoma. Int. J. Cancer 2007, 120, 769-775.

21. Cadron, I.; van Gorp, T.; Amant, F.; Vergote, I.; Moerman, P.; Waelkens, E.; Daemen, A.; van de Plas, R.; de Moor, B.; Zeillinger, R. The use of laser microdissection and SELDI-TOF MS in ovarian cancer tissue to identify protein profiles. Anticancer Res. 2009, 29, 1039-1045.

22. Gagne, J.P.; Ethier, C.; Gagne, P.; Mercier, G.; Bonicalzi, M.E.; Mes-Masson, A.M.; Droit, A.; Winstall, E.; Isabelle, M.; Poirier, G.G. Comparative proteome analysis of human epithelial ovarian cancer. Proteome Sci. 2007, 5, e16.

23. Ikeda, K.; Monden, T.; Kanoh, T.; Tsujie, M.; Izawa, H.; Haba, A.; Ohnishi, T.; Sekimoto, M.; Tomita, N.; Shiozaki, H.; et al. Extraction and analysis of diagnostically useful proteins from formalin-fixed, paraffin-embedded tissue sections. J. Histochem. Cytochem. 1998, 46, 397-403.

24. Rodriguez-Rigueiro, T.; Valladares-Ayerbes, M.; Haz-Conde, M.; Blanco, M.; Aparicio, G.; Fernandez-Puente, P.; Blanco, F.J.; Lorenzo, M.J.; Aparicio, L.A.; Figueroa, A. A novel procedure for protein extraction from formalin-fixed paraffin-embedded tissues. Proteomics 2011, $11,2555-2559$.

25. Gustafsson, J.O.; Oehler, M.K.; McColl, S.R.; Hoffmann, P. Citric acid antigen retrieval (CAAR) for tryptic peptide imaging directly on archived formalin-fixed paraffin-embedded tissue. J. Proteome Res. 2010, 9, 4315-4328.

26. Ostasiewicz, P.; Zielinska, D.F.; Mann, M.; Wisniewski, J.R. Proteome, phosphoproteome, and $\mathrm{N}$-glycoproteome are quantitatively preserved in formalin-fixed paraffin-embedded tissue and analyzable by high-resolution mass spectrometry. J. Proteome Res. 2010, 9, 3688-3700. 
27. Magrane, M. UniProt Knowledgebase: A Hub of Integrated Protein Data. Available online: http://www.ebi.ac.uk/integr8 (accessed on 21 March 2013).

28. Keller, A.; Nesvizhskii, A.I.; Kolker, E.; Aebersold, R. Empirical statistical model to estimate the accuracy of peptide identifications made by MS/MS and database search. Anal. Chem. 2002, 74, 5383-5392.

29. Keller, A.; Eng, J.; Zhang, N.; Li, X.J.; Aebersold, R. A uniform proteomics MS/MS analysis platform utilizing open XML file formats. Mol. Syst. Biol. 2005, 1, e2005.0017.

30. Liu, H.; Sadygov, R.G.; Yates, J.R., 3rd. A model for random sampling and estimation of relative protein abundance in shotgun proteomics. Anal. Chem. 2004, 76, 4193-4201.

31. Hood, B.L.; Conrads, T.P.; Veenstra, T.D. Mass spectrometric analysis of formalin-fixed paraffin-embedded tissue: Unlocking the proteome within. Proteomics 2006, 6, 4106-4114.

32. Hood, B.L.; Conrads, T.P.; Veenstra, T.D. Unravelling the proteome of formalin-fixed paraffin-embedded tissue. Brief. Funct. Genomic. Proteomic. 2006, 5, 169-175.

33. Hood, B.L.; Grahovac, J.; Flint, M.S.; Sun, M.; Charro, N.; Becker, D.; Wells, A.; Conrads, T.P. Proteomic analysis of laser microdissected melanoma cells from skin organ cultures. J. Proteome Res. 2010, 9, 3656-3663.

34. Flanagan, M.B.; Dabbs, D.J.; Brufsky, A.M.; Beriwal, S.; Bhargava, R. Histopathologic variables predict Oncotype DX recurrence score. Mod. Pathol. 2008, 21, 1255-1261.

35. McCarty, K.S., Jr.; Miller, L.S.; Cox, E.B.; Konrath, J.; McCarty, K.S.S. Estrogen receptor analyses. Correlation of biochemical and immunohistochemical methods using monoclonal antireceptor antibodies. Arch. Pathol. Lab. Med. 1985, 109, 716-721.

36. Ding, L.; Ellis, M.J.; Li, S.; Larson, D.E.; Chen, K.; Wallis, J.W.; Harris, C.C.; McLellan, M.D.; Fulton, R.S.; Fulton, L.L.; et al. Genome remodelling in a basal-like breast cancer metastasis and xenograft. Nature 2010, 464, 999-1005.

37. Campbell, P.J.; Yachida, S.; Mudie, L.J.; Stephens, P.J.; Pleasance, E.D.; Stebbings, L.A.; Morsberger, L.A.; Latimer, C.; McLaren, S.; Lin, M.L.; et al. The patterns and dynamics of genomic instability in metastatic pancreatic cancer. Nature 2010, 467, 1109-1113.

38. Chung, K.H.; Lee, D.H.; Kim, Y.; Kim, T.H.; Huh, J.H.; Chung, S.G.; Lee, S.; Lee, C.; Ko, J.J.; An, H.J. Proteomic identification of overexpressed PRDX 1 and its clinical implications in ovarian carcinoma. J. Proteome Res. 2010, 9, 451-457.

39. Basu, A.; Banerjee, H.; Rojas, H.; Martinez, S.R.; Roy, S.; Jia, Z.; Lilly, M.B.; de Leon, M.; Casiano, C.A. Differential expression of peroxiredoxins in prostate cancer: Consistent upregulation of PRDX3 and PRDX4. Prostate 2011, 71, 755-765.

40. Canet, B.; Pons, C.; Espinosa, I.; Prat, J. Ovarian clear cell carcinomas: RHO GTPases may contribute to explain their singular biologic behavior. Hum. Pathol. 2011, 42, 833-839.

41. Gadducci, A.; Guerrieri, M.E.; Riccardo, G.A. New insights on the pathogenesis of ovarian carcinoma: Molecular basis and clinical implications. Gynecol. Endocrinol. 2012, 28, 582-586.

42. Lalwani, N.; Prasad, S.R.; Vikram, R.; Shanbhogue, A.K.; Huettner, P.C.; Fasih, N. Histologic, molecular, and cytogenetic features of ovarian cancers: Implications for diagnosis and treatment. Radiographics 2011, 31, 625-646. 
43. Shih, I.; Panuganti, P.K.; Kuo, K.T.; Mao, T.L.; Kuhn, E.; Jones, S.; Velculescu, V.E.; Kurman, R.J.; Wang, T.L. Somatic mutations of PPP2R1A in ovarian and uterine carcinomas. Am. J. Pathol. 2011, 178, 1442-1447.

44. McConechy, M.K.; Anglesio, M.S.; Kalloger, S.E.; Yang, W.; Senz, J.; Chow, C.; Heravi-Moussavi, A.; Morin, G.B.; Mes-Masson, A.M.; Australian Ovarian Cancer Study Group; et al. Subtype-specific mutation of PPP2R1A in endometrial and ovarian carcinomas. J. Pathol. 2011, 223, 567-573.

(C) 2013 by the authors; licensee MDPI, Basel, Switzerland. This article is an open access article distributed under the terms and conditions of the Creative Commons Attribution license (http://creativecommons.org/licenses/by/3.0/). 